\title{
In Vitro Evaluation of Topical Microemulsion of Capsaicin Free of Surfactant
}

\author{
Pengwei Zhang, ${ }^{a}$ Wenyuan GaO, ${ }^{*, a}$ Li Zhang, ${ }^{b}$ Li Chen, ${ }^{b}$ Qufei Shen,,${ }^{b}$ Xiaohui Wang,,${ }^{b}$ and \\ Ying $\mathrm{CuI}^{b}$ \\ ${ }^{a}$ School of Pharmaceutical Science and Technology, Tianjin University; Tianjin 300072, P. R. China: and ${ }^{b}$ Department of \\ Pharmaceutics, Medical College of Chinese People's Armed Police Force; Tianjin 300162, P. R. China. \\ Received April 24, 2008; accepted August 25, 2008; published online September 22, 2008
}

\begin{abstract}
Topical microemulsion of capsaicin without surfactant was developed in this study. In these systems, the oil phase was benzyl alcohol, and the cosurfactant was propylene glycol and ethanol. The drop-size of the systems was measured by dynamic light scattering method in order to distinguish true solution from microemulsion. The transdermal performance of the microemulsions was evaluated in vitro by Franz diffusion cells fitted with rat skins. The results showed the drop-size of the microemulsions without surfactant was smaller than that of the systems with Tween 80 and the permeation rate of capsaicin decreased as the content of Tween 80 increased. In the system composed of water, benzyl alcohol and propylene glycol, the permeation rate increased with the enhancement of benzyl alcohol and water. But water content had little effect on the permeation rate in the microemulsions with ethanol as cosurfactant.
\end{abstract}

Key words microemulsion; capsaicin; topical application; benzyl alcohol

Capsaicin (8-methyl $N$-vanillyl-6 nonenamide), an active compound extracted from Chilli peppers, is widely used for treatment of rheumatoid arthritis, osteoarthritis, diabetic neuropathy and postherpetic neuralgia. ${ }^{1)}$ However, first pass effect of capsaicin is very high, ${ }^{2)}$ and the half-life of capsaicin after intravenous administration is only $7.06 \mathrm{~min}$ in rats ${ }^{3)}$ and 12.44 min in rabbits. ${ }^{4)}$ Adverse events of capsaicin mainly include burning, stinging and erythema at the application site. ${ }^{5)}$ Topical application of this drug can avoid the first-pass metabolism. Moreover, due to the long period of treatment cycle, ${ }^{5)}$ topical therapeutical systems (TTS) is very suitable for capsaicin.

Microemulsion is a single optically isotropic and thermodynamically stable liquid mixture of oil, water, surfactant and cosurfactant. Due to its advantages, for example, easy making, good stability, enhancing skin permeation and protecting drug against oxidation, ${ }^{6}$ ) microemulsion has become a potential TTS for both hydrophilic and hydrophobic drugs. ${ }^{7}$ The structure of microemulsion consists of a disordered, connected surfactant monolayer separating the two solvent domains. It is "balanced" in the sense that this monolayer sheet has no preference in curving toward either of the two solvents. ${ }^{8}$ The conditions of microemulsion formation are as follows: (1) very low interfacial tension at the water-oil interface; (2) a highly fluid interfacial surfactant film; (3) the penetration and association of the molecules of the oil phase with the interfacial surfactant film. ${ }^{9}$ ) However, in a microemulsion system, there is commonly a high ratio of surfactants in the formulation, therefore, they may affect the skin barrier function and cause potential risks of irritation and toxicity in long-term application. ${ }^{10,11)}$ Furthermore, some researches have demonstrated that the increased concentration of surfactant can depress the permeation rate of drugs. ${ }^{12-15)}$ This article was intended to investigate the potential of a new microemulsion formulation without surfactant for topical drug delivery of capsaicin. Surfactant-free microemulsions were prepared in other fields, ${ }^{16-18)}$ but were not used in topical pharmaceuticals. The novel microemul- sion was based on benzyl alcohol and short-chain alcohol. Benzyl alcohol is a potential transdermal enhancer and has an amphiphilic molecular structure, ${ }^{19)}$ which is helpful for the formation of microemulsion, ${ }^{16)}$ and can be used as oil phase in microemulsions. ${ }^{20,21)}$ Furthermore, benzyl alcohol is a commonly used drug for local anesthesia and alleviating itching, thus, it may be helpful to reduce the side-effects of capsaicin.

\section{MATERIALS AND METHODS}

Materials Capsaicin was purchased from Nanjing Tianshu Bioengineering Co., Ltd. (Nanjing, China). Benzyl alcohol (BA), propylene glycol (PG), ethanol (EA) and $n$-butanol were obtained from Tianjin 1st Chemical Agent Factory. Tween 80 (Polysorbate 80) was purchased from Shanghai Chemical Co., Ltd. Water was double distilled in a quartz inferior-boiling apparatus. Methanol was HPLC grade. The other chemicals were analytical grade or pharmaceutical grade complied with 2005 Chinese pharmacopoeia standard.

Construction of Phase Diagrams In order to determine the concentration range of components for the existing range of microemulsions, ternary phase diagrams were constructed using water titration method at room temperature $\left(25^{\circ} \mathrm{C}\right)$. For each phase diagram, the ratios of oil to cosurfactant were varied as $1: 9,2: 8,3: 7,4: 6,5: 5,6: 4,7: 3,8: 2,9: 1$, or $10: 0$. Water was added drop-wisely into the mixture stirred by a magnetical stirrer to reach equilibrium quickly. The phase boundary was determined by observing the changes of the sample appearance being from turbid to transparent or from transparent to turbid. The content of each component in solutions was derived from precise mass measurement. No attempt was made to distinguish among oil-in-water, waterin-oil or bicontinuous type microemulsions.

Preparation of Microemulsion, Cream and Hydrogel of Capsaicin After the microemulsion regions in the phase diagrams were determined, the microemulsion formulations were selected at different component ratios as described in 
Table 1. The Composition of Microemulsion Formulations

\begin{tabular}{|c|c|c|c|c|c|c|}
\hline Composition & $\begin{array}{l}\text { Water } \\
(\%)\end{array}$ & $\begin{array}{c}\text { Benzyl alcohol } \\
(\%)\end{array}$ & $\begin{array}{l}\text { Propylene glycol } \\
\qquad(\%)\end{array}$ & $\begin{array}{c}\text { Ethanol } \\
(\%)\end{array}$ & $\begin{array}{c}\text { Tween } 80 \\
(\%)\end{array}$ & $\begin{array}{c}\text { Capsaicin } \\
(\%)\end{array}$ \\
\hline F1 & 25 & 5 & 70 & & & 0.075 \\
\hline $\mathrm{F} 2$ & 25 & 15 & 60 & & & 0.075 \\
\hline F3 & 25 & 35 & 40 & & & 0.075 \\
\hline F4 & 25 & 45 & 30 & & & 0.075 \\
\hline F5 & 10 & 42 & 48 & & & 0.075 \\
\hline F6 & 40 & 28 & 32 & & & 0.075 \\
\hline F7 & 25 & 35 & & 40 & & 0.075 \\
\hline F8 & 25 & 45 & & 30 & & 0.075 \\
\hline F9 & 40 & 28 & & 32 & & 0.075 \\
\hline F10 & 25 & 35 & 13.3 & & 26.7 & 0.075 \\
\hline F11 & 25 & 35 & 20 & & 20 & 0.075 \\
\hline F12 & 25 & 35 & 26.7 & & 13.3 & 0.075 \\
\hline F13 & & 100 & & & & 0.075 \\
\hline F14 & & & 100 & & & 0.075 \\
\hline F15 & & & & 100 & & 0.075 \\
\hline
\end{tabular}

Table 1. In order to prepare the drug loaded microemulsions, measured capsaicin was dissolved in benzyl alcohol. Then the cosurfactant was mixed with the oil and water was added to the oil phase. The mixture was stirred by a magnetical stirrer for $10 \mathrm{~min}$.

The capsaicin cream was prepared as follows. ${ }^{22)}$ Stearic acid $12.0 \mathrm{~g}$, lanolin $2.0 \mathrm{~g}$, liquid paraffin $14.0 \mathrm{~g}$ and stearyl alcohol $1.0 \mathrm{~g}$ were melt in $80^{\circ} \mathrm{C}$ water bath and $75 \mathrm{mg}$ of capsaicin was dissolved in the mixture to form a uniform oil phase. The mixture of glycerine $5.0 \mathrm{~g}$, trolamine $2.0 \mathrm{~g}$ and water $64.0 \mathrm{~g}$ was heated to $80^{\circ} \mathrm{C}$ as the water phase. With agitation the water phase was added to the oil phase slowly and kept stirring until the cream dropped to room temperature.

The capsaicin hydrogel was prepared as the formulation F15 containing 6\% carboxymethyl cellulose sodium salt according to the study by Wang et al. ${ }^{23)}$

The microemulsion, cream and hydrogel formulations were used to perform in vitro experiments after $24 \mathrm{~h}$ of preparation. In all of the formulations, the content of capsaicin was $0.075 \%(\mathrm{w} / \mathrm{w})$.

Characterization and Stability of Microemulsions The droplet size distribution of the microemulsions was measured by dynamic light scattering (DLS) using a Zetasizer HS3000 instrument (Malvern Instruments, England). The measurement was performed by backscatter at a fixed angle $\left(90^{\circ}\right)$ at $25^{\circ} \mathrm{C}$.

The electrical conductivity was determined by DDS-307 conductivity meter (Shanghai Leici Instrument Co., China). All of above measurements were carried out at $25^{\circ} \mathrm{C}$.

The centrifuge tests were carried out to assess the physical stability of microemulsion. Microemulsion vehicles were centrifuged for $30 \mathrm{~min}$ at $13000 \mathrm{rpm}$ and for $4 \mathrm{~h}$ at $4000 \mathrm{rpm}$ in the centrifuge test. ${ }^{24)}$ Microemulsions were sealed in ampoules and stored at $-4{ }^{\circ} \mathrm{C}$ and $80^{\circ} \mathrm{C}$ for $10 \mathrm{~d}$. Then the concentration of capsaicin was measured to judge the chemical stability.

In Vitro Skin Permeation Study The abdominal skins were obtained from Sprague-Dawley rats (males, 200$300 \mathrm{~g}$ ). After hairs were removed with an electric clipper (Walh, U.S.A.), the skins were excised. The extraneous tissues were trimmed surgically and the subcutaneous fat was wiped off with isopropyl alcohol. Then the skins were washed off isopropyl alcohol with water and examined for integrity. The permeation experiments were performed using Franz diffusion cells. The skins were clamped between the donor and the receptor chambers of vertical diffusion cells. The cell had an effective diffusion area of $2.83 \mathrm{~cm}^{2}$ and a $7 \mathrm{ml}$ receptor volume, and filled with ethanol-pH 7.4 PBS $(1: 1, \mathrm{v} / \mathrm{v})^{23)}$ maintained temperature at $37^{\circ} \mathrm{C}$. The formulations $(2.0 \mathrm{ml}$ or $2 \mathrm{~g})$ containing capsaicin were gently placed in the donor chamber. At 2, 3, 4, 6, 8, 10 and $12 \mathrm{~h}$, all of the solution in the receptor chambers was withdrawn for HPLC determination and the chambers were immediately refilled with fresh solution. Each sample was performed in triplicate. The cumulative amount $\left(Q_{t}\right)$ was calculated from the following equation:

$$
Q_{t}=V_{t} C_{t}+\sum_{i=0}^{t-1} V_{\mathrm{s}} C_{i}
$$

where $C_{t}$ is the drug concentration of the receptor solution at each sampling time, $C_{i}$ is the drug concentration of the $i$ th sample, and $V_{t}$ and $V_{\mathrm{s}}$ are the volume of the receptor solution and the sample, respectively. Data were expressed as the cumulative capsaicin permeation per unit of skin surface area, $Q_{t} / S\left(\mu \mathrm{g} \cdot \mathrm{cm}^{-2}\right)$.

The permeation rate of capsaicin at steady-state $\left(J_{S}\right.$, $\mu \mathrm{g} \cdot \mathrm{cm}^{-2} \cdot \mathrm{h}^{-1}$ ) through rat skin was calculated from the slope of linear portion of the cumulative amount permeated through the rat skins per unit area versus time plot. ${ }^{24)}$

HPLC Analysis of Capsaicin Samples were analyzed by a modified HPLC method described previously. ${ }^{25)}$ The analytical column is Shimadzu ODS VP $(5 \mu \mathrm{m}, 4.6 \times 25 \mathrm{~cm})$. The mobile phase is methanol-water- $\mathrm{H}_{3} \mathrm{PO}_{4}(85: 15: 0.1)$ at the flow rate of $1.0 \mathrm{ml} / \mathrm{min}$. The validation of analytical method was made prior to the experiments.

\section{RESULTS AND DISCUSSION}

Phase Studies Cosurfactant can decrease interfacial tension between oil and water in microemulsion and adjust the flexibility of interfacial membrane, which is an important factor for the formation of microemulsion without surfactant. 

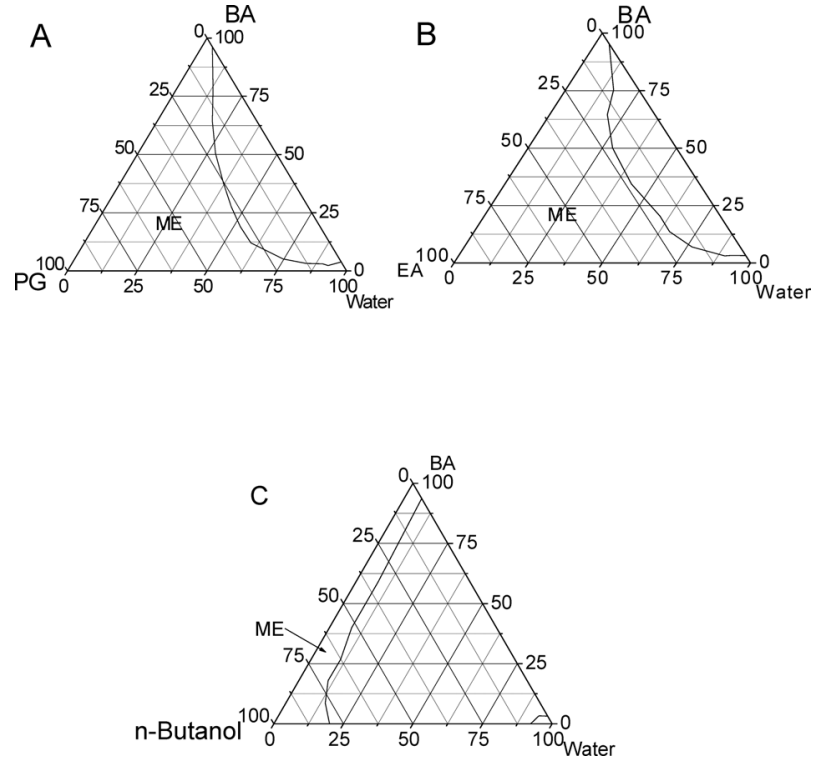

Fig. 1. Ternary Phase Diagrams of Microemulsion Composed of Benzyl Alcohol, Water and Different Cosurfactant (A) Propylene Alcohol; (B) Ethanol; (C) $n$-Butanol

ME, microemulsion.

This study chose short-chain alcohols as cosurfactants. ${ }^{15,26)}$ Phase diagrams were used to determine the concentration range of components for the existence range of microemulsions. The ternary phase diagrams with various cosurfactants were shown in Fig. 1. The transparent microemulsion region was presented in phase diagrams. No distinct conversion from water-in-oil (w/o) to oil-in-water $(\mathrm{o} / \mathrm{w})$ microemulsions was observed. The diagrams of microemulsion including propylene glycol had isotropic region, which was similar to that of ethanol. The order of the addition did not change the physicochemical properties of the microemulsions. The area of microemulsion using $n$-butanol as cosurfactant was much smaller compared to propylene glycol and ethanol. It may be because propylene glycol and ethanol are miscible with benzyl alcohol and water, and can distribute in the oil and the water phase, then decrease the interfacial energy between the two phases. However, the solubility of $n$-butanol in water is smaller than that in propylene glycol and ethanol, and it cannot effectively reduce the tension of interface between oil and water.

Characterization and Stability of Microemulsion Study In order to discriminate the true solution from microemulsion of the systems, the droplet size of systems was measured by DLS and the results were summarized in Table 2 . The F4 and F8 containing 30\% cosurfactant, propylene glycol and ethanol, respectively, had the lowest average droplet size. For propylene glycol and ethanol, the average droplet size decreased as the oil increased. On the contrary, it increased with the increased amount of Tween 80 . With propylene alcohol and ethanol as cosurfactant, there was not very distinct difference between their droplet sizes in a similar systems. It was surprising that when the ratio of oil was below $15 \%$ using propylene glycol as cosurfactant, the droplet size detected was much bigger than $760 \mathrm{~nm}$, the wavelength of visible light; however, the systems were clear, thermodynamically stable and isotropic mixtures with all the
Table 2. The Droplet Size of the Microemulsions and Skin Permeation Rate of Capsaicin

\begin{tabular}{rrrcc}
\hline \hline Composition & $\begin{array}{c}\text { Diameters } \\
(\mathrm{nm})\end{array}$ & Polydispersity & $\begin{array}{c}\text { Conductivity } \\
\left(\mu \mathrm{s} \cdot \mathrm{cm}^{-1}\right)\end{array}$ & $\begin{array}{c}J_{s} \\
\left(\mu \mathrm{g} \cdot \mathrm{cm}^{-2} \cdot \mathrm{h}^{-1}\right)\end{array}$ \\
\hline Cream & - & - & - & $2.78 \pm 0.19^{\Delta \Delta}$ \\
Hydrogel & - & - & - & $7.35 \pm 0.57^{* *}$ \\
F1 & $2856 \pm 332$ & 0.195 & 0.667 & $2.59 \pm 0.40^{\Delta \Delta}$ \\
F2 & $1450 \pm 166$ & 0.232 & 1.229 & $5.96 \pm 1.05^{* *, \Delta \Delta}$ \\
F3 & $12.6 \pm 2.5$ & 0.267 & 2.76 & $12.92 \pm 1.02^{* *, \Delta \Delta}$ \\
F4 & $6.6 \pm 1.0$ & 0.301 & 3.14 & $13.83 \pm 1.02^{* *, \Delta \Delta}$ \\
F5 & - & - & 1.33 & $9.79 \pm 0.75^{* *, \Delta \Delta}$ \\
F6 & $13.7 \pm 2.3$ & 0.234 & 3.21 & $14.50 \pm 1.12^{* *, \Delta \Delta}$ \\
F7 & $8.3 \pm 2.0$ & 0.223 & 3.63 & $10.78 \pm 2.11^{* *, \Delta \Delta}$ \\
F8 & $7.8 \pm 1.3$ & 0.269 & 4.40 & $11.11 \pm 1.43^{* *, \Delta \Delta}$ \\
F9 & $12.6 \pm 3.5$ & 0.365 & 4.06 & $12.63 \pm 1.71^{* *, \Delta \Delta}$ \\
F10 & $16.6 \pm 3.7$ & 0.277 & 72.6 & $8.45 \pm 1.45^{* *, \Delta \Delta}$ \\
F11 & $27.7 \pm 5.5$ & 0.256 & 89.2 & $10.07 \pm 1.82^{* *, \Delta \Delta}$ \\
F12 & $30.8 \pm 8.5$ & 0.200 & 71.4 & $12.16 \pm 2.25^{* *, \Delta \Delta}$ \\
F13 & - & - & - & $5.16 \pm 0.76^{* *, \Delta}$ \\
F14 & - & - & - & $0.55 \pm 0.19^{* *, \Delta \Delta}$ \\
F15 & - & - & - & $2.13 \pm 0.22^{\Delta \Delta}$ \\
\hline
\end{tabular}

** $p<0.01$ compared with the cream; $\Delta p<0.05, \Delta \Delta p<0.01$ compared with the hydrogel.

characteristics of microemulsion. Such phenomenon was also discovered by previous studies. ${ }^{16,27)}$ In the systems with low oil fraction, there is a high ratio of propylene glycol, and this relatively high ratio of cosurfactant could distribute into the oil phase, which causes the oil droplet similar to amphiphilic particles. In a dynamic equilibrium course, these droplets are incorporated into the outer phase and also dispersed as inner phase. They are dynamic systems in which the interface is continuously and spontaneously fluctuating. ${ }^{28)}$ The high radius can be explained as a temporal, apparent and aggregated radius. ${ }^{27)}$ In an o/w system, the phenyl groups of benzyl alcohol aggregate together and the groups of $-\mathrm{CH}_{2} \mathrm{OH}$ are arrayed toward the outer phase. But the water molecules aggregate at the centre combined with the group of $-\mathrm{CH}_{2} \mathrm{OH}$ in a w/o system. The droplet size of the system with $10 \%$ water, $42.8 \%$ oil and $47.2 \%$ cosurfactant could not be detected out. The water molecule is smaller than that of benzyl alcohol and propylene glycol. In the low water fraction system, the water molecules are uniformly dispersed in the system linking with hydroxyl group of benzyl alcohol and propylene glycol by hydrogen bond, and the mixture become a true solution system. ${ }^{29}$ The molecular weight of Tween 80 is much bigger than that of benzyl alcohol and it can form a stable film at the interface of the two phases. Such a droplet can load more oil or water, which leads to a bigger dropsize of the microemulsions.

In all of the microemulsions without surfactant, the conductivity was much smaller than that of the systems with Tween 80 .

The centrifuge tests showed that all microemulsion had good physical stability. However, the phase separation was observed when vehicles stored at $-4^{\circ} \mathrm{C}$. But they recovered clear after shaking gently when they stored in room temperature for a few minutes. The capsaicin concentration did not change significantly after the microemulsions were heated in $80^{\circ} \mathrm{C}$ for $10 \mathrm{~d}$.

In Vitro Skin Permeation Studies Due to the narrow region of microemulsion with $n$-butanol as cosurfactant, only 


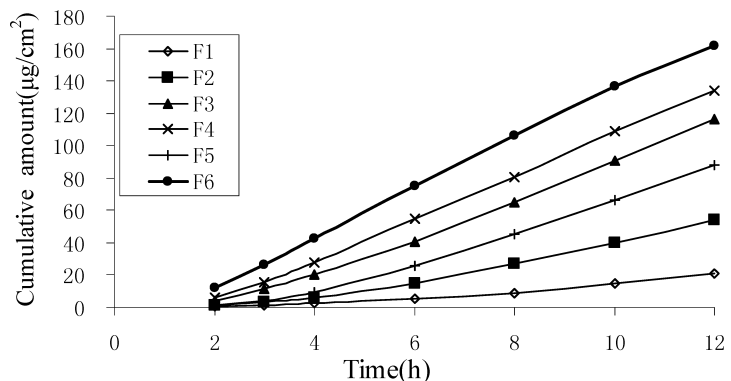

Fig. 2. Permeation Profiles of Capsaicin through Excised Rat Skin from Microemulsions Containing Different Contents of Benzyl Alcohol $(n=3)$

propylene glycol and ethanol were studied. These two alcohols are lowly irritant and toxic and they have been widely used as permeation enhancer for many drugs. ${ }^{30-32)}$ The steady-state flux of the tested formulations is presented in Table 2. The permeation profiles of capsaicin through rat skins from different formulations were shown from Figs. 2 to 5. A steady increased permeation of capsaicin with time was shown. The permeation profiles of the microemulsions followed zero order release kinetics. Most of the steady-state fluxes of microemulsions were higher than those of the capsaicin cream, hydrogel and single solvent $(t$-test, $p<0.05)$. Figure 2 showed that the enhancement of ratio of benzyl alcohol to propylene glycol led to the rise of permeation rate of capsaicin through the rat skin. F5 and F6 were arrayed on the line of $\mathrm{F} 3$ to water $100 \%$ and symmetrically at the two side of the line of $25 \%$ water in the phase diagram. So the ratio of benzyl alcohol to propylene glycol was fixed at 0.875 . When the percentage of the concentration of water was $40 \%$, the permeation rate was the highest. It may be because water can hydrate skin and cause the corneocytes to swell. Moreover, there are some lacunar domains in the stratum corneum embedded within the lipid bilayers. Under normal physiological conditions, these lacunae are scattered and discontinuous. Under condition of high concentration of water, the lacunae expand, interconnect and form a continuous "pore pathway". The formation of such a route would markedly enhance drug penetration. ${ }^{33}$ Therefore, with the increasing amount of water in the system, the transdermal permeation rate of drug was improved, which is consistent with the previous studies. ${ }^{15,34)}$ However, the content of benzyl alcohol and water are contradictive factors between each other. Microemulsion systems with more benzyl alcohol can accept little water and vice versa.

When using ethanol as cosurfactant, the permeation rate deceased slightly with the increase of ratio of benzyl alcohol to ethanol (ANOVA test, $p<0.05$ ) and the water concentration had little effect on the skin permeation (Fig. 3). The results suggested that propylene glycol and ethanol have different mechanisms in penetration enhancement.

Tween 80 and propylene glycol are commonly used in the preparation of microemulsions. Propylene glycol has higher boiling point than ethanol which makes the microemulsion more stable. So the effect of Tween 80 with propylene glycol on the permeation rate was studied in this paper. The result was shown in Fig. 4. The skin permeation rate of capsaicin decreased from $12.16 \pm 2.25$ to $8.45 \pm 1.45 \mu \mathrm{g} \cdot \mathrm{cm}^{-2} \cdot \mathrm{h}^{-1}$ as the ratio of surfactant to cosurfactant increased from $1: 2$ to $2: 1$ (ANOVA test, $p>0.05$ ). This finding was confirmed by

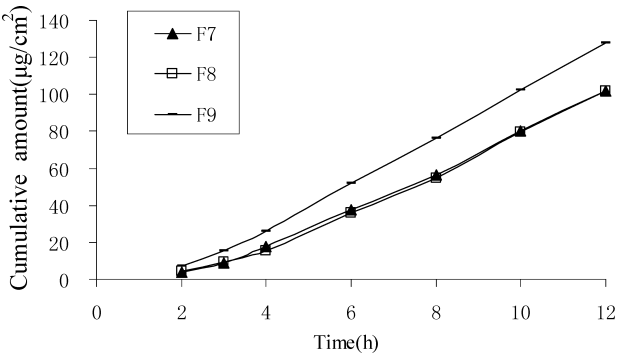

Fig. 3. Permeation Profiles of Capsaicin through Excised Rat Skin from Microemulsions Containing Different Contents of Ethanol $(n=3)$

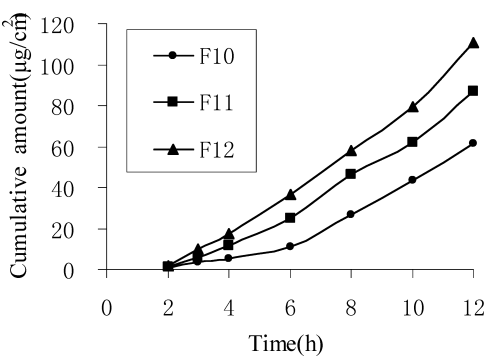

Fig. 4. Permeation Profiles of Capsaicin through Excised Rat Skin from Microemulsions Containing Different Contents of Tween $80(n=3)$

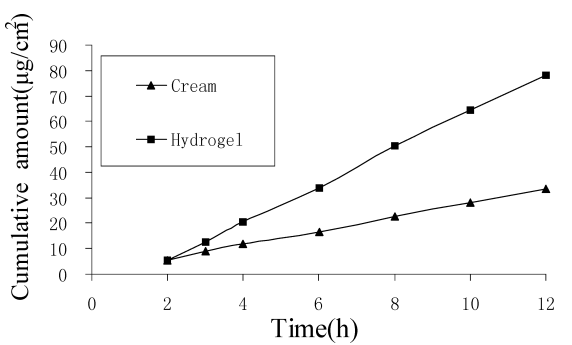

Fig. 5. Permeation Profiles of Capsaicin through Excised Rat Skin from Capsaicin Cream and Hydrogel $(n=3)$

previous studies which showed when the content of surfactant decreased, the skin permeation rate of drug increased. ${ }^{13-15}$ The explanation may be that all of the studied drugs are lipophilic and their affinity to oil is greater than to water. As described previously, in the microemulsion, more surfactant can form steady film and load more oil in the droplets. This causes a decreased thermodynamic activity of the drug in microemulsion at the higher content of surfactant. ${ }^{15)}$ The thermodynamic activity of drug in the formulation is an important driving force for the release and penetration of the drug into skin. The thermodynamic driving force for release reflects the relative activities of the drug in different phases. ${ }^{14)}$

\section{CONCLUSION}

A microemulsion system free of surfactants was investigated as a potential drug delivery systems for capsaicin. The microemulsion regions in the ternary systems composed of benzyl alcohol, water and propylene glycol, ethanol or $n$-butanol were characterized. Phase diagrams showed that propylene glycol and ethanol are potential cosurfactants to prepare surfactant-free microemulsion. DLS measurement can distinguish the systems from true solution and microemulsion. The dropsize of the microemulsions increases with the increase of 
Tween 80 , but the in vitro permeation rate decreases with it. Higher content of benzyl alcohol and water leads to a higher permeation rate of capsaicin. In vitro permeation studies showed that microemulsions without surfactants have higher skin permeation rates than those of the cream, hydrogel and single solvent used in these experiments.

\section{REFERENCES}

1) Fusco B. M., Giacovazzo M., Drugs, 53, 909—914 (1997).

2) Donnerer J., Amann R., Schuligoi R., Lembeck F., Naunyn-Schmiedebergs Arch. Pharmacol., 342, 357-361 (1990).

3) Kawada T., Watanabe T., Katsura K., Takami H., Iwai K., J. Chromatogr., 329, 99-105 (1985).

4) Fang J. Y., Wu P. C., Huang Y. B., Tsai Y. H., Int. J. Pharm., 128, $169-177$ (1996).

5) Mason L., Moore R. A., Derry S., Edwards J. E., McQuary H. J., Br. Med. J., 328, 991-994 (2004).

6) Spiclin P., Gasperlin M., Kmetec V., Int. J. Pharm., 222, 271-279 (2001).

7) Kogan A., Garti N., Adv. Colloid Interface, 123, 369-385 (2006).

8) Daicic J., Olsson U., Wennerström H., Langmuir, 11, 2451-2458 (1995).

9) Kreilgaard M., Adv. Drug Deliv. Rev., 54, S77-S98 (2002).

10) López A., Linares F., Cortell C., Herröez M., Int. J. Pharm., 202, $133-140$ (2000).

11) Wilhelm K. P., Bottjer B., Siegers C. P., Br. J. Dermatol., 145, 709715 (2001).

12) Ktistis G., Niopas I., J. Pharm. Pharmacol., 50, 413-418 (1998).

13) Rhee Y. S., Choi J. G., Park E. S., Chi S. C., Int. J. Pharm., 228, 161170 (2001).

14) Chen H., Chang X., Weng T., Zhao X., Gao Z., Yang Y., Xu H., Yang
X., J. Controlled Release, 98, 427-436 (2004).

15) Yuan Y., Li S., Mo F., Zhong D., Int. J. Pharm., 321, 117-123 (2006).

16) Kamogawa K., Matsumoto M., Kobayashi T., Langmuir, 15, 1913 1917 (1999).

17) Smith G. D., Donelan C. E., Barden R. E., J. Colloid Interface Sci., 60, 488-496 (1977).

18) Zoumpanioti M., Karali M., Xenakis A., Stamatis H., Enzyme Microb. Technol., 39, 531-539 (2006).

19) Nimni M. E., Ertl D., Oakes R. A., J. Pharm. Pharmacol., 42, 729 731 (1990).

20) Boltri L., Morel S., Trotta M., J. Pharm. Belg., 49, 315-320 (1994).

21) Trotta M., Morel S., Gasco M. R., Pharmazie, 52, 50-53 (1997).

22) Liu K. C., Liu C. H., Wang X. M., Dang L., Song G. Y., Shandong Sci., 17, 33-36 (2004).

23) Wang Y. Y., Hong C. T., Chiu W. T., Fang J. Y., Int. J. Pharm., 224, 89-104 (2001).

24) Zhao X., Liu J. P., Zhang X. L., Int. J. Pharm., 327, 58-64 (2006).

25) Magnusson B. M., Koskinen L. O. D., Int. J. Pharm., 195, 55-62 (2000).

26) Attwood D., Mallon C., Taylor C. J., Int. J. Pharm., 84, R5-R8 (1992).

27) Baroli B., López-Quintela M. A., Delgado-Charro M. B., Fadda A. M., Blanco-Méndez J. B., J. Controlled Release, 69, 209-218 (2000).

28) Lam A. C., Schechter R. S., J. Colloid Interface Sci., 120, 56-63 (1987).

29) Chatenay D., Urbach W., Cazabat A. M., Langevin D., Phys. Rev. Lett., 54, 2253-2256 (1985).

30) Gao S., Singh J., J. Controlled Release, 51, 193-199 (1998).

31) Arellano A., Santoyo S., Martin C., Ygartua P., Eur. J. Pharm. Sci., 7 , $129-135$ (1998).

32) Lee P. J., Langer R., Shastri V. P., Pharm. Res., 20, 264-269 (2003).

33) Elias P. M., Tsai J., Menon G. K., JID Symp. Proc., 7, 79-85 (2002).

34) Alvarez-Figueroa M. J., Blanco-Mendez J., Int. J. Pharm., 215, 57-65 (2001). 